\title{
Erratum to: Anatomy of the proximal femur at the time of total hip arthroplasty is a matter of morphotype and etiology but not gender
}

\author{
Matthieu Ollivier · Sebastien Parratte • \\ Thomas Le Corroller • Andrea Reggiori • \\ Pierre Champsaur $\cdot$ Jean-Noël Argenson
}

Published online: 25 September 2014

(C) Springer-Verlag France 2014

\section{Erratum to: Surg Radiol Anat}

\section{DOI 10.1007/s00276-014-1368-5}

Owing to an unfortunate oversight the third author name has been incorrectly published as Lecorroller $\mathrm{T}$. However the author name should read as Le Corroller T.

The online version of the original article can be found under doi:10.1007/s00276-014-1368-5.

M. Ollivier · S. Parratte - T. Le Corroller · A. Reggiori ·

P. Champsaur · J.-N. Argenson

Aix-Marseille University, CNRS, ISM UMR 7287,

13284 Marseille, France

M. Ollivier · S. Parratte · A. Reggiori · J.-N. Argenson Orthopedic Surgery Department, APHM, Hôpital Sainte Marguerite, Institute for Locomotion, 13009 Marseille, France

M. Ollivier · T. Le Corroller · P. Champsaur Laboratoire d'anatomie de La Faculté de Médecine de Marseille, 13005 Marseille, France

M. Ollivier ( $\square)$

Institut du mouvement et de l'appareil locomoteur, Service de Chirurgie Orthopédique, Hôpital Sainte-Marguerite, 270,

Boulevard Sainte-Marguerite, BP 29-13274, Marseille Cedex 09,

France

e-mail: ollivier.mt@gmail.com

T. Le Corroller · P. Champsaur

Radiology Department, APHM, Hôpital Sainte Marguerite,

Institute for Locomotion, 13009 Marseille, France 